\title{
Peer review: a flawed but essential process
}

\author{
${ }^{1} \mathrm{~J}$ Iredale, ${ }^{2} \mathrm{R}$ Al-Shahi Salman \\ ${ }^{1}$ Professor of Medicine, Dean of Clinical Medicine and Director of MRC Centre for Inflammation Research, University of Edinburgh; ${ }^{2} M R C$ \\ Senior Clinical Fellow, Honorary Consultant Neurologist, University of Edinburgh, Edinburgh, UK
}

KEYWORDS Medline, Cochrane Review, post hoc review, meta-analysis, continuing professional development (CPD)

DECLARATION OF INTERESTS Professor Iredale was Associate Editor of Hepatology from 2006-II

\author{
Correspondence to J Iredale, \\ Queen's Medical \\ Research Institute, \\ 47 Little France Crescent \\ Edinburgh EHI 6 4TJ, UK \\ tel. +44 (0) I3 I 2426686
e-mail (PA)
carolynn.walthew@ed.ac.uk
}

Four times a year the mail delivery brings us a transient heart-sink moment. The heart-sink is precipitated by the arrival of a pile of Fellowship applications from the Wellcome Trust. Invariably though, the moment is transient; for once the envelope is opened and reviewing begins in earnest we become absorbed in a world sometimes familiar, oftentimes unfamiliar, of new scientific ideas, exciting new diagnostic technologies, translational initiatives and clinical trials of familiar drugs and new compounds. Always interesting and sometimes challenging, reviewing these applications prompts activity on Medline, background research and stimulates thoughts about one's own research and practice.

Of course, research grants are only one small part of the document reviewing that doctors undertake as part and parcel of their professional life. The scientific value of medical journals has traditionally depended on the rigour with which peer review of submitted articles is undertaken. With the advent of open access publication, other 'metrics' will speak for the quality and newsworthiness of papers but, for either model, editors and journals depend on the willingness of colleagues to peer review research papers, editorials and opinion pieces and offer in turn a valued judgement on their substance, quality and importance. Thus peer review is absolutely critical to maintaining the highest standards in published evidence within our current publishing models.

Peer review as a rigid standard expected of our journals, editorial policies and grant reviewing bodies however has not been without significant criticism. For example for a single research paper it is easy to argue that two opinions, even if expert, cannot be completely exhaustive. A recent Cochrane Review concluded that the quality of evidence available to support an uncompromising scientific view of the value of peer review in improving the quality of biomedical research doesn't exist.' None of us has an absolutely exhaustive knowledge and reviewers will fail to identify flaws from time to time. Moreover, the reviewers may provide flawed or poor quality reviews, which may be particularly susceptible to bias from undeclared competing interests when the peer review process is anonymous. However, viewed as an activity engaged in over the long-term, peer reviewing has a significant educational potential for any individual doctor. Of course, to work, this system requires reviewers to be open and honest about their own shortcomings with respect to specific areas of analysis, but in our experience such opinion is welcomed by editors and drives a positive debate and discussion about the merits of a study.

Although the other models of publishing and post hoc review facilitated by internet-based publication are growing in importance and role, it's interesting that a significant tranche of mainstream medical and scientific journals and research grant funding bodies still adhere to a traditional model of peer review. One might reasonably conclude that despite its deficiencies, peer review in this context could be described rather like Churchill's view of democracy ('Democracy is the worst form of government, except for all those other forms that have been tried from time to time') and represents the 'least worst' process.

It is easy to criticise peer review, but we believe a more constructive approach would be to enhance the process, quality and experience of review by initiating measures such as those suggested, summarised in Table I.

TABLE I Enhancing the process, quality and experience of peer review

Recognise the educational value of peer review

- Award CME points for undertaking review

- Endorse it as CPD in job planning

Enhance the educational yield of peer review

- Make reference to research methodology standards (www.equator-network.org)

- Journals must feedback other reviewers' comments and editorial decision once a decision has been made.

- Do research to increase the quality of peer review 
Few would argue with the concept that research and analysis, data gathering (either as a primary research project or for a meta-analysis or Cochrane Review), data assimilation, conclusion, presentation and communication are invaluable as educational exercises for the medical student, junior doctor, general practitioner (GP), principal or consultant. But what of the educational value of the process of peer review itself? Our view, based on personal experience, is that the careful review of papers, with an appropriate and detailed analysis of methodology, logic and the quality and rigour of the work is also highly educational. This is especially so when review is undertaken with reference to accepted methodological standards (e.g. Preferred Reporting Items for Systematic Reviews and Meta-Analysis [PRISMA] for systematic reviews, Statement for Reporting of Diagnostic Accuracy/Quality Assessment of Diagnostic Accuracy Studies [STARD/QUADAS] for diagnostic tests, Consolidated Standards of Reporting Trials [CONSORT] for trials, and Strengthening the Reporting of Observational Studies in Epidemiology [STROBE] for observational studies. More information on good reporting of health research studies can be found on the Equator website: www.equator-network.org).

Moreover, there are clear and direct educational advantages to be gained from participating in peer review. The process of reviewing papers submitted to a journal invariably involves an initial review undertaken by (usually) two individuals in isolation. Following this they submit their critique to the editor, together with (if requested) their recommendation with respect to dispensation for publication, with an accompanying rationale highlighting any changes and work by which the paper could be improved. Sometimes, reviewers and/or editors request that a further opinion is sought from another specialist reviewer such as a statistician. Indeed many journals will, as a matter of course, seek statistical advice on each paper. Once this information is amassed and complete, the editorial team make a decision. Although not common practice to all journals, for major periodicals, the editorial decision and any supporting rationale together with the reviews and advice provided by all parties, is shared with the peer reviewers at the time that the manuscript authors receive the editorial disposition and the peer review. Sadly, this practice is not uniform; the value of peer review is diminished by the failure of some journals to adopt this approach. We believe this failing also sends the wrong message out to the reviewing community. If the process of peer review is to maximise its educational value then it is self-evident that reviewers must receive feedback (in the form of the disposition and further independent reviews). To offer the reviewer nothing in our opinion is both discourteous and of no educational value.

Furthermore, the process described above provides the specialist statistical or trial design expert with the opportunity to read a balanced opinion on the scientific merits and the importance of a particular study and its critical references generated by those with perhaps a clear understanding of the underlying biology.

Reviewing remains a component of continuing professional development (CPD) for physicians and in our opinion it is imperative that doctors are encouraged to contribute to the review process throughout their career. Until recently journals like Neurology, for example, recognised and awarded continuing medical education (CME) credit to reviewers but this was recently discontinued because the programme did not comply with the Accreditation Council for Continuing Medical Education (ACCME) guidelines. ${ }^{2}$ Any moves to diminish the value and impact of reviewing, in CPD assessments and job plans, should be viewed with concern.

Despite its deficiencies and failings, peer review remains an important tool in the screening and assessment of research and development on which practice and service change are based. A wide ranging 'College' of reviewers with respect to a specialty interest, research activity and experience from all specialties is essential to maintaining and developing the standards of UK medicine. We should do all that we can to ensure we create a culture in which this activity is supported and nurtured.

\section{REFERENCES}

I Jefferson T, Rudin M, Brodney Folse S et al. Editorial peer review for improving the quality of reports of biomedical studies. Cochrane Database Syst Rev 2007; 2:MR0000I6.

2 Neurology.org [Internet]. CME for peer reviewers. Baltimore: American Academy of Neurology [cited 2012 Sept 25]. Available from: http://www.neurology.org/site/misc/info_review.xhtml 